\title{
Complexes of Block Copolymers in Solution: Tree Approximation
}

\author{
Bernard J. Geurts ${ }^{1}$ and Ruud van Damme ${ }^{2}$
}

Received April 5, 1989; revision July 17, 1989

\begin{abstract}
We determine the statistical properties of block copolymer complexes in solution. These complexes are assumed to have the topological structure of (i) a tree or of (ii) a line-dressed tree. In case the structure is that of a tree, the system is shown to undergo a gelation transition at sufficiently high polymer concentration. However, if the structure is that of a line-dressed tree, this transition is absent. Hence, we show the assumption about the topological structure to be relevant for the statistical properties of the system. We determine the average size of the complexes and calculate the viscosity of the system under the assumption that the complexes geometrically can be treated as porous spheres.
\end{abstract}

KEY WORDS: Block copolymers; generating function; Polya's theorem; gelation.

\section{INTRODUCTION}

In this paper we consider the statistical physics of a system of linear, two-component block copolymers in solution. Schematically these block copolymers have the structure ABA. The solvent is rather poor for the end groups (A), which are much smaller than the connecting flexible polymers (B), for which the solvent is quite good. As a consequence of this, the end groups tend to cluster together in so-called "domains," connected by B parts of the block copolymers. In this way, larger complexes consisting of domains, containing a certain number of A parts connected by B parts, are formed. We use the methods of equilibrium statistical physics to analyze the statistical properties of these complexes. The problem of finding these

\footnotetext{
${ }^{1}$ Center for Theoretical Physics, Twente University, 7500 AE Enschede, The Netherlands.

${ }^{2}$ Department of Applied Mathematics, Twente University, 7500 AE Enschede, The Netherlands.
} 
properties is related to the counting of the number of topologically different complexes that can be formed with $k$ polymers. This is solved in detail for complexes which have the topology of a tree. We derive the size distribution of the complexes and show that at sufficiently high concentration the system undergoes a transition from a liquid phase to a gel phase. The average number of polymers per complex is determined as a function of polymer concentration. Finally, the viscosity as a function of polymer concentration, below the gel transition, is determined under the assumption that the complexes can geometrically be treated as porous spheres. We present results both for normal trees as well as for line-dressed trees, in which every line in the treelike structure corresponds to an arbitrary number of connecting B parts. The most apparent difference between these two classes of structures is that if the complexes have the topology of line-dressed trees, the gelation transition is absent. The restriction to treelike structures implies that we do not take cycles and loops in a systematic way into account. We extend the treatment to cover a large class of structures with loops and cycles in a subsequent paper. ${ }^{(1)}$

Block copolymers have been studied experimentally quite extensively in the past. It is known that these block copolymers in a solvent as described above organize in such a way that complexes are formed containing domains connected by the B parts of the polymers. In equilibrium there is a continuous addition and removal of $\mathrm{A}$ parts to and from domains; the complexes formed not only continuously change shape, but also topological structure, i.e., the way in which the connections between different parts of a complex are organized. At sufficiently high concentration and under suitable conditions such systems may show a transition from a liquid phase to a phase in which a macroscopic gel is formed. Using the methods of statistical physics, one can "translate" the study of these systems to one of counting the number of complexes of a given size. This counting problem can be treated once the topological structure of the complexes is agreed upon. In this paper we consider both normal treelike structures as well as line-dressed trees, and use the generating function method as described by Harary and Palmer ${ }^{(2)}$ to count the number of structures containing a certain number of polymers. In Fig. 1 we show how a complex is mapped onto the corresponding graph; the domains are mapped onto the vertices of the graph and the connecting polymers are identified with the edges of the graph. The number of $\mathrm{A}$ parts in a domain thus corresponds to the functionality of the vertex associated with it. In a study of the statistical physics of star molecules formed from block copolymers of structure AB we determined the average number of molecules in a star. ${ }^{(3)}$ This gives an order-ofmagnitude estimate for the average functionality of the vertices in the block copolymer complexes considered in this paper. 

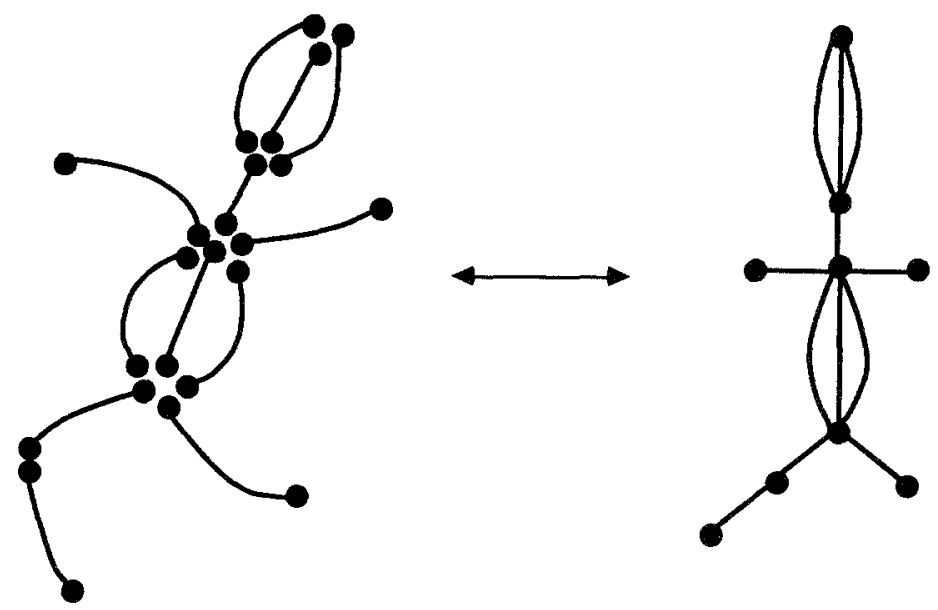

Fig. 1. Association of a block copolymer complex with its corresponding graph. The domains are mapped onto the vertices and the B parts are identified with the edges.

In Section 2 we derive the configuration sum for this system. Then, in Section 3 we treat in detail the counting problem one faces in order to evaluate this configuration sum explicitly for the case that the complexes have the topological structure of a normal tree. The number of complexes of a given size is counted using the generating function method as described by Harary and Palmer, ${ }^{(2)}$ based on the central theorem of Polya. The asymptotic behavior of the size distribution of the complexes is found in Section 4, in which we also determine the average number of polymers per complex as a function of polymer density and show the gelation transition in the system. In Section 5 we extend the treatment to cover the case in which the complexes have the topological structure of line-dressed trees. Finally, in Section 6 we express the viscosity of the system in terms of the size distribution of the complexes under the assumption that the complexes can be treated as porous spheres. We compare predictions for the viscosity of the two cases treated.

\section{THE CONFIGURATION SUM OF A SYSTEM OF BLOCK COPOLYMERS IN SOLUTION}

In this section we first derive the configuration sum of the system considered and then determine the equilibrium size distribution for the number of complexes containing $k$ polymers.

The calculation of the configuration sum involves several steps. Consider a large volume $V$ in which there are $N(\gg 1)$ block copolymers in solution at thermodynamic equilibrium at temperature $T$. Let $\gamma_{k}$ denote 
the number of complexes containing $k$ molecules and $\Gamma \equiv\left(\gamma_{1}, \gamma_{2}, \ldots\right)$ denote a macrostate of the system. Such a macrostate must be compatible, i.e.,

$$
\sum_{k=1}^{N} k \gamma_{k}=N
$$

The configuration sum $Q(\Gamma)$ can be expressed as

$$
Q(\Gamma)=\frac{1}{N !} \Omega(\Gamma) Z(\Gamma)
$$

where $\Omega(\Gamma)$ is the number of microstates compatible with a given macrostate $\Gamma$ and $Z(\Gamma)$ is the configuration sum for a specific distribution of the complexes. The number of microstates $\Omega(\Gamma)$ is equal to the number of different ways in which $N$ indistinguishable molecules can be put together such that there are exactly $\gamma_{k}$ complexes containing $k$ polymers. It is given by

$$
\Omega(\Gamma)=N !\left(\prod_{k=1}^{N}\left(\gamma_{k} ! k !^{\gamma k}\right)\right)^{-1}
$$

Each complex containing $k$ polymers can be moved around through the volume $V$, leading to a combinatorial factor $v_{k} \equiv V / V_{k}$, in which $V_{k}$ is the average volume of such a complex. This average volume in principle not only depends on $k$, but also on the specific way in which the polymers are organized in the complex. However, since we assumed the B parts to be much larger than the A parts, this volume is mainly determined by the number of polymers in the complex; the way these polymers are connected is of less importance. Finally, all permutations of the polymers within a complex must be included and we obtain

$$
Z(\Gamma)=\prod_{k=1}^{N}\left(v_{k} k ! Q_{k}\right)^{\gamma_{k}}
$$

where $Q_{k}$ is the configuration sum of a complex, containing $k$ polymers, which is at a fixed position in space and the polymers within the complex are in a specific permutation. Combining the last three equations gives

$$
Q(\Gamma)=\prod_{k=1}^{N}\left(\gamma_{k} !\right)^{-1}\left(v_{k} Q_{k}\right)^{\gamma_{k}}
$$

We will now specify $Q_{k}$ in more detail.

The complexes consist of domains which are connected by the B parts of the molecules. If we concentrate on complexes containing $k$ molecules, 
one notices that there is a large number of topologically different ways of organizing the molecules in such complexes. The number of domains $m$, as well as the functionality of the domains, i.e., the number of A parts grouped together in a domain, can be chosen from a large number of options. Let $\left\{n_{j}\right\}$ denote the functionality vector of a complex, i.e., there are $n_{j}$ domains of functionality $j ; j=1,2, \ldots, 2 k$. The weight factor associated with a domain of functionality $j$ will be deoted by $g_{j}$. Next, we must associate a weight factor with the connecting $\mathbf{B}$ parts. As is well known, ${ }^{(4)}$ the weight factor for the $\mathbf{B}$ parts in a complex with $k$ polymers, $h_{k}$, can be written as $h_{k} \sim \mu^{M k} M^{a_{k}-1}$, where $\mu$ is a constant, $M$ is the number of segments of the $\mathrm{B}$ parts, and $a_{k}$ is a coefficient depending on the number of polymers in the complex. Since we assumed $M \gg 1$, we only keep the dominant term, i.e., we put $h_{k}=h^{k} ; h \sim \mu^{M}$. Thus, we may write

$$
Q_{k}=h^{k} \sum_{m=1}^{k+1} \sum_{\left\{n_{j}\right\}} T_{k, m,\left\{n_{j}\right\}} \prod_{j=1}^{2 k} g_{j}^{n_{j}}
$$

Here, $T_{k, m,\{n\}}$ is the number of complexes containing $k$ molecules grouped together such that there are $m$ domains whose functionality vector is $\left\{n_{j}\right\}$. The summation over all $\left\{n_{j}\right\}$ vectors is subject to the obvious constraints

$$
\begin{gathered}
\sum_{j=1}^{2 k} j n_{j}=2 k \\
\sum_{j=1}^{2 k} n_{j}=m
\end{gathered}
$$

The upper limit $(k+1)$ in the summation over $m$ in (2.6) can be inferred from the fact that the maximal number of domains is obtained for the complex in which all molecules are grouped in a fashion not containing loops or cycles.

As a final specification of the model, we let $E_{j}$ denote the cluster energy of a domain containing $j \mathrm{~A}$ parts. We suppose that adding an extra A part to an already existing domain yields a constant energy gain, independent of the size of the domain. Hence, we write

$$
E_{j}=U_{0}-(j-1) U_{1}
$$

which implies

$$
g_{j}=e^{-E_{j} / k T} \equiv g_{1} \alpha^{j-1}
$$

where $\alpha=\exp \left(U_{1} / k T\right)$. Since the A parts tend to cluster, one has 
$0<U_{0}<U_{1}$. Combination of $(2.6)-(2.8)$ and (2.10) then yields for the configuration sum

$$
Q(\Gamma)=\prod_{k=1}^{N}\left(\gamma_{k} !\right)^{-1}\left(v_{k} h^{k} \alpha^{2 k}\right)^{\gamma_{k}}\left(\sum_{m=1}^{k+1} T_{k, m} \xi^{m}\right)^{\gamma_{k}}
$$

where $\xi=g_{1} / \alpha<1$ and $T_{k, m}$ is the number of complexes containing $k$ molecules and $m$ domains.

The equilibrium distribution of the complexes can be found by maximizing $Q(\Gamma)$ subject to the constraint (2.1). Introducing a Lagrange multiplier $\lambda$ for this constraint, one finds that this problem is equivalent to finding the unconstrained maximum of

$$
\mathscr{L}(\Gamma)=\ln [Q(\Gamma)]-\lambda \sum_{k=1}^{N} k \gamma_{k}
$$

Using Stirling's approximation [i.e., $\ln (x !)=x \ln (x)-x$ ], one obtains for the equilibrium distribution $\Gamma^{*}$, by equating $\partial \mathscr{L} / \partial \gamma_{k}=0$ for all $k$,

$$
\gamma_{k}^{*}=v_{k}\left(h \alpha^{2} e^{-\lambda}\right)^{k} \sum_{m=1}^{k+1} T_{k, m} \xi^{m}
$$

and the requirement that the macrostate $\Gamma^{*}$ must satisfy (2.1) leads to an equation from which $\lambda$ can be determined. Solving this yields the size distribution explicitly and various statistical properties can be calculated straightforwardly.

We notice that the statistical properties of the complexes can be found explicitly once the numbers $T_{k, m}$ are determined. This counting problem can, however, only be treated once the topological structure of the complexes is agreed upon. This will form the subject of the next section, in which we assume the complexes to have the topology of a tree. The extension to line-dressed trees is treated in Section 2.

\section{COUNTING THE NUMBER OF COMPLEXES IN THE TREE APPROXIMATION}

In the previous section we translated the problem of determining the statistical properties of the block copolymer complexes to the problem of counting the number of topologically different complexes that can be formed with $k$ polymers grouped such that there are $m$ domains. We will treat this problem for the case that the complexes have the topology of a tree and use the generating function method as described by Harary and Palmer. ${ }^{(2)}$ This assumption about the topological structure implies that we 
disregard loops and cycles. The line-dressed tree case constitutes a first extension in which simple cycles are taken into account (cf. Section 5). In a subsequent paper ${ }^{(1)}$ we extend the treatment and include loops and cycles in a systematic way.

We will derive functional equations for the generating functions determining the number of complexes in the tree approximation. It will prove to be expedient to consider rooted trees first, i.e., trees in which one of the vertices is assigned to be the "base point." The generating function for unrooted trees will be expressed in terms of the generating function for rooted trees afterward. Then we consider the singular behavior of the generating functions in order to obtain the asymptotic behavior of the number of trees with a specified number of edges.

A block copolymer complex contains a certain number of domains (vertices) connected by $\mathrm{B}$ parts of the polymers (edges). Let $x$ denote the counting variable for edges and $y$ for vertices; then we define the generating function for rooted trees as

$$
T_{R}(x, y)=y+\sum_{k=1}^{\infty} \sum_{m=2}^{k+1} T_{k, m}^{R} x^{k} y^{m}
$$

Thus, $T_{k, m}^{R}$ is the number of rooted trees with $k$ edges and $m$ vertices. For complexes having the topology of a tree, we notice that a tree with $k$ edges contains exactly $k+1$ vertices, i.e., $T_{k, m}^{R}=0$ if $m \neq k+1$. We derive a functional equation for $T_{R}(x, y)$.

In Fig. 2 we sketch the diagram for rooted trees. It expresses the fact that a rooted tree is either a single vertex or a vertex from which an arbitrary number of edges emanate connecting to a rooted tree. There is a vast literature on these counting problems (see the book of Harary and Palmer ${ }^{(2)}$ and references therein). The main difficulty is in avoiding the double counting of trees and incorporating the symmetries, since all edges correspond to mutually indistinguishable polymers (hence, we cannot use the methods developed by Wiegel and Perelson ${ }^{(5)}$ ). This problem is beauti-

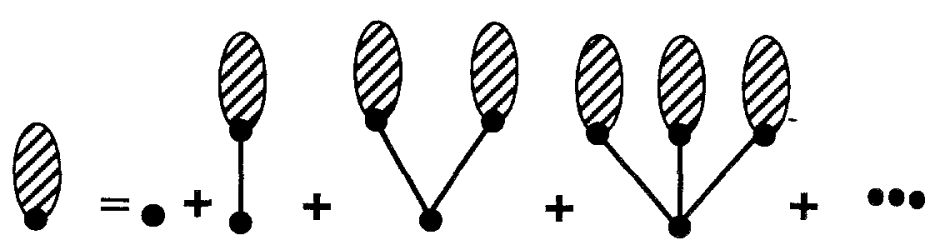

Fig. 2. Diagram for rooted trees (hatched blob with a full dot) in which we show that a rooted tree is a vertex from which an arbitrary number of edges emanate connecting to a rooted tree. 
fully taken care of in the central theorem of Polya. For rooted trees one notices that any permutation of the branches emanating from the root results in equivalent trees, i.e., are to be identified. Let $S_{l}$ be the symmetric permutation group on $l$ objects, i.e., the group of all permutations on $l$ elements. One must count the diagrams in Fig. 2 taking care of the permutation identification, i.e., $S_{l}$. Let $T_{P}(x, y) \equiv x T_{R}(x, y)$, that is, the generating function for planted trees, i.e., rooted trees rooted at an end point. The correct way to count the number of rooted trees is to use the cycle index over $S_{l}$ of $T_{P}(x, y),{ }^{(2)}$ and we have, from the diagram in Fig. 2,

$$
\begin{aligned}
T_{R}(x, y) & =y+y Z\left(S_{1}, T_{P}\right)+y Z\left(S_{2}, T_{P}\right)+y Z\left(S_{3}, T_{P}\right)+\cdots \\
& =y \sum_{l=0}^{\infty} Z\left(S_{l}, T_{P}\right)
\end{aligned}
$$

in which $Z\left(S_{l}, f(x, y)\right)$ is the cycle index over the symmetric group $S_{l}$ acting on the function $f(x, y)$. It is a polynomial in the variables $f\left(x^{n}, y^{n}\right)$, $n=1,2, \ldots, l$, and expresses the above-mentioned permutation identification of the branches. For an exact treatment, details, and definition of the cycle index we refer the reader to Chapter 2 of the book of Harary and Palmer. ${ }^{(2)}$ A central property of $Z\left(S_{l}, f\right)$ is

$$
\sum_{l=0}^{\infty} Z\left(S_{l}, f(x, y)\right)=\exp \left(\sum_{l=1}^{\infty} \frac{T_{P}\left(x^{l}, y^{l}\right)}{l}\right)
$$

and combination of this with (3.2) together with the definition of $T_{P}(x, y)$ implies that $T_{P}$ satisfies the functional equation

$$
T_{P}(x, y)=x y \exp \left(\sum_{l=1}^{\infty} \frac{T_{P}\left(x^{l}, y^{l}\right)}{l}\right)
$$

Hence, $T_{P}$ is a function of $x y$ and the first few terms are found from (3.4) using the symbolic manipulation program REDUCE, ${ }^{(6)}$

$$
\begin{aligned}
T_{P}(x, y)= & \sum_{k=1}^{\infty} T_{k, k}^{P} x^{k} y^{k} \\
= & x y+(x y)^{2}+2(x y)^{3}+4(x y)^{4}+9(x y)^{5} \\
& +20(x y)^{6}+48(x y)^{7}+115(x y)^{8}+286(x y)^{9}+\cdots
\end{aligned}
$$

The number of rooted trees follows from (3.5), since $T_{k, k+1}^{R}=T_{k+1, k+1}^{P}$; $k=0,1,2, \ldots$. In spite of the fact that in principle every coefficient in the generating function can be calculated using (3.4), it is not possible to express these numbers in closed form. We will consider the asymptotic 
dependence of these numbers on $k$ momentarily and first turn to the generating function for unrooted trees.

Since the block copolymer complexes in the tree approximation correspond to trees which do not have roots, we must still unroot the trees counted by $T_{R}(x, y)$. Let $T(x, y)$ denote the generating function for unrooted trees, i.e.,

$$
T(x, y)=y+\sum_{k=1}^{\infty} T_{k, k+1} x^{k} y^{k+1}
$$

where $T_{k, k+1}$ is the number of unrooted trees with $k$ edges. We derive an expression for $T(x, y)$ in terms of $T_{R}(x, y)$. In Fig. 3 we have drawn all rooted and unrooted trees with up to three edges. Notice that the number of unrooted trees with $k$ edges is always smaller than or equal to the number of corresponding rooted trees. Hence, the number of rooted trees has to be corrected in order to yield the number of unrooted trees. We will describe this correction in detail next.

In Fig. 4 we have drawn two examples of unrooted trees. The tree in Fig. 4a has six nonequivalent vertices which could be used as roots for corresponding rooted trees that would all be counted as different. So this unrooted tree would be counted six times if we considered rooted trees. Likewise, there are five nonequivalent, nonsymmetry edges, that is, edges which connect nonequivalent vertices. So, if we would consider all different "line-rooted" trees resulting from the diagram in Fig. 4a, we would be able to make five different ones. Hence, for trees with no symmetry edges, such

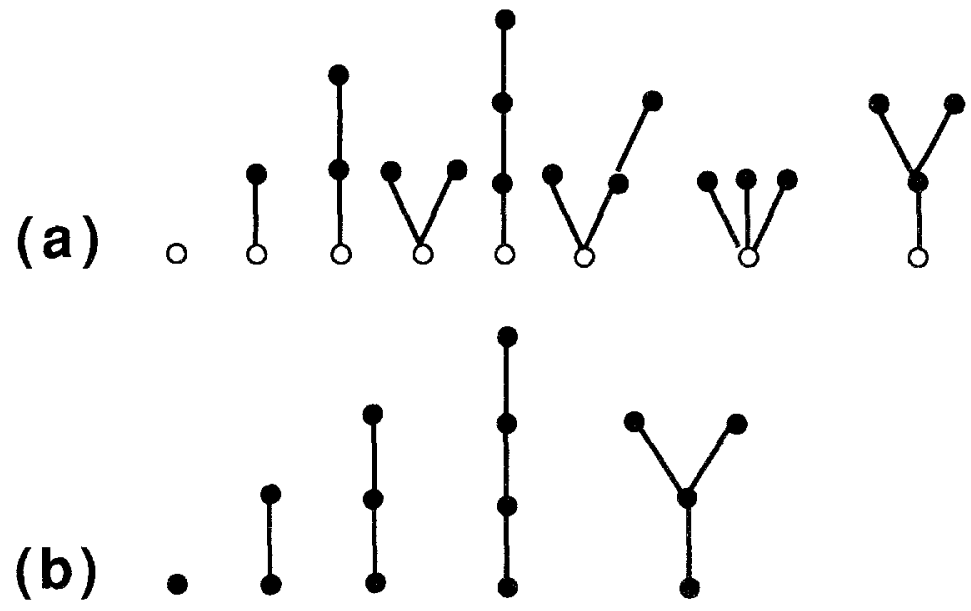

Fig. 3. Diagram showing all (a) rooted and (b) unrooted trees with up to three edges. 


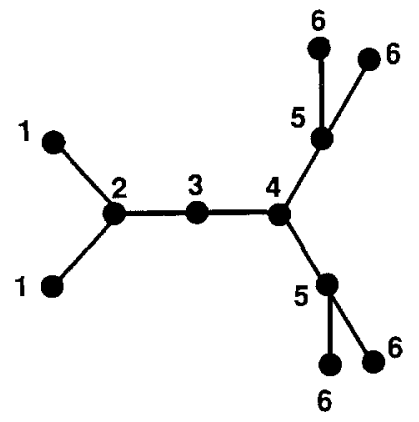

(a)

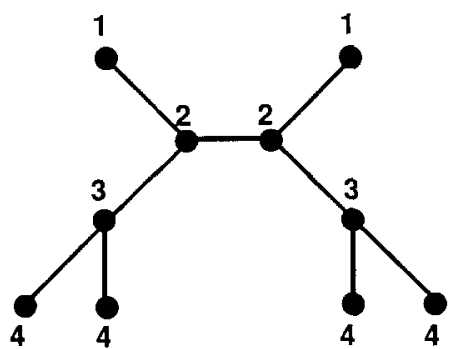

(b)

Fig. 4. Two examples of larger trees. (a) An asymmetric, (b) a symmetric case. The different nonequivalent points are labeled.

as in Fig. 4a, we must subtract the number of nonsymmetry line-rooted trees from the number of rooted trees to get the number of unrooted trees. In Fig. $4 b$ we have drawn a symmetric tree with four nonequivalent vertices, three nonequivalent nonsymmetry edges, and one symmetry line. Again, the same conclusion holds that the number of unrooted trees is equal to the number of rooted trees minus the number of line-rooted trees rooted at a nonsymmetry line. ${ }^{(7)}$

So the generating function $T(x, y)$ can be expressed as

$$
T(x, y)=T_{R}(x, y)-L(x, y)
$$

where $L(x, y)$ is the generating function for line-rooted trees rooted at a nonsymmetry line. A line-rooted tree can be generated by connecting the roots of two rooted trees. The connecting edge is a nonsymmetry line if the two rooted trees connected are different. Hence, $L(x, y)$ equals the generating function for line-rooted trees minus the generating function for line-rooted trees rooted at a symmetry line. The generating function for line-rooted trees is obviously given by $x T_{R}^{2}(x, y) / 2$, since it is generated by connecting (a factor $x$ ) two rooted trees [a factor $T_{R}^{2}(x, y)$ ]. The factor $1 / 2$ appears since we must identify permutations of the two rooted trees connected. Next, a root line is a symmetry line if we connect two identical rooted trees. So, the generating function for symmetry-line rooted trees is given by $x T_{R}\left(x^{2}, y^{2}\right) / 2$. The factor $T_{R}\left(x^{2}, y^{2}\right)$ expresses the fact that we connect two identical rooted trees, hence the doubling of the number of edges and vertices $\left(x^{2}\right.$ and $\left.y^{2}\right)$. Thus, we find for $T(x, y)$ the equation ${ }^{(7)}$

$$
T(x, y)=T_{R}(x, y)-\frac{1}{2} x\left[T_{R}^{2}(x, y)-T_{R}\left(x^{2}, y^{2}\right)\right]
$$


Inserting $T_{R}(x, y)$ into this, we find, using REDUCE, for the first few terms

$$
\begin{aligned}
T(x, y)= & y+x y^{2}+x^{2} y^{3}+2 x^{3} y^{4}+3 x^{4} y^{5}+6 x^{5} y^{6} \\
& +11 x^{6} y^{7}+23 x^{7} y^{8}+47 x^{8} y^{9}+\cdots
\end{aligned}
$$

It is left to the reader to verify the first few terms using Fig. 3.

We next study the singular behavior of the generating functions to obtain the asymptotic behavior of the number of trees with $k$ edges. We study $T_{P}$ first and then consider the consequences for $T_{R}$ and $T$. In the following we keep $0<y \leqslant 1$ and $y$ fixed. The power series expansion (3.5) for $T_{P}$ has a convergence radius $R(y)$ given by

$$
R(y)=\lim _{k \rightarrow \infty} \frac{T_{k, k}^{P} y^{k}}{T_{k+1 . k+1}^{P} y^{k+1}}=R(1) y^{-1}
$$

Hence, $T_{P}(x, y)$ is an analytic function of $x$ if $|x|<R(y)$, and it has a singularity at $x=R(y)$. Consider the function

$$
\mathscr{F}(\zeta, x, y)=x y \exp \left(\zeta+\sum_{l=2}^{\infty} \frac{T_{P}\left(x^{l}, y^{l}\right)}{l}\right)-\zeta
$$

Then $\mathscr{F}\left(T_{P}(x, y), x, y\right)=0$, and using the implicit function theorem, we can expand $T_{P}(x, y)$ in a Taylor series around any $(x, y)$ unless

$$
\mathscr{F}_{\zeta}\left(T_{P}(x, y), x, y\right)=T_{P}(x, y)-1=0
$$

Hence, at the singularity, $T_{P}(R(y), y)=1$. Since the second derivative of $\mathscr{F}$ with respect to $\zeta$ has the property that $\mathscr{F}_{\zeta \zeta}\left(T_{P}(R(y), y), R(y), y\right)=1$, the generating function $T_{p}$ can be expanded as ${ }^{(2)}$

$$
\begin{aligned}
T_{P}(x, y)= & 1-b_{1}(y)[R(y)-x]^{1 / 2} \\
& +b_{2}(y)[R(y)-x]+b_{3}(y)[R(y)-x]^{3 / 2}+\cdots
\end{aligned}
$$

for $x$ close to but smaller than $R(y)$. It is straightforward to show that the functions $b_{1}(y)$ and $b_{2}(y)$ are given by

$$
\begin{aligned}
\frac{1}{2} b_{1}^{2}(y) & =\lim _{x \uparrow R(y)} \frac{\mathscr{F}_{x}\left(T_{P}(x, y), x, y\right)}{\mathscr{F}_{\zeta \zeta}\left(T_{P}(x, y), x, y\right)} \\
& =\frac{1}{R(y)}\left[1+\sum_{l=2}^{\infty} R^{l}(y) \partial_{x} T_{P}\left(R^{l}(y), y^{l}\right)\right]
\end{aligned}
$$

and

$$
b_{2}(y)=\frac{1}{3} b_{1}^{2}(y)
$$


Since $T_{P}(x, y)$ has the property

$$
R^{l}(y) \partial_{x} T_{P}\left(R^{l}(y), y\right)=R^{l}(1) \partial_{x} T_{P}\left(R^{l}(1), 1\right)
$$

which can be verified using (3.5), we may rewrite this as

$$
b_{1}(y)=b_{1}(1) y^{1 / 2} ; \quad b_{2}(y)=\frac{1}{3} b_{1}^{2}(1) y
$$

As a consequence of the square root singularity in $T_{P}(x, y)$, it follows that asymptotically

$$
T_{k, k}^{P} y^{k} \approx \frac{b_{1}(1) R^{1 / 2}(1)}{2 \sqrt{\pi}} R^{-k}(1) k^{-3 / 2} y^{k}
$$

showing that $T_{k, k}^{P} \sim R^{-k}(1) k^{-3 / 2}$.

We will now estimate $R(1)$ and $b_{1}(1)$ in order to completely specify the asymptotic behavior, and consider the consequences for unrooted trees afterward. It is not possible to express $R(1)$ and $b_{1}(1)$ in closed form. Rather, we will derive precise upper and lower bounds for these quantities.

We turn to $R(1)$ first and notice that, since $T_{P}(R(1), 1)=1$, we may write, in view of (3.4),

$$
R(1)=e^{-1} \prod_{l=2}^{\infty} e^{-T_{P, l} / l}
$$

in which we put $T_{P, l} \equiv T_{P}\left(R^{l}(1), 1\right)$ for notational convenience. Notice that if we derive upper and lower bounds for all $T_{P, l}, l=2,3, \ldots$, we obtain upper and lower bounds for $R(1)$. In the Appendix we prove that

$$
T_{P, l}<e^{-l}
$$

and hence

$$
\begin{aligned}
R(1)>R_{-}(1) & \equiv e^{-1} \exp \left(-\sum_{i=2}^{\infty} \frac{1}{l} e^{-1}\right) \\
& =\frac{e-1}{e} \exp \left(\frac{1-e}{e}\right)=0.335949 \ldots
\end{aligned}
$$

Refering to the Appendix, we also have the lower bound

$$
T_{P, l}>R_{-}^{l}(1)
$$

and hence, after some calculation,

$$
R(1)<R_{+}(1) \equiv\left[1-R_{-}(1)\right] e^{R_{-}(1)-1}=0.341828 \ldots
$$


For this particular case, $R(1)$ has been determined by Otter. ${ }^{(7)} \mathrm{He}$ estimated $R(1)=0.3383219 \ldots$; so our upper and lower bounds are very accurate. This is of importance as well for the line-dressed tree case, for which no results are available in literature. In subsequent calculations we will use $R(1)=\left[R_{-}(1)+R_{+}(1)\right] / 2=0.33888 \ldots$, which is a good approximation of the value given by Otter.

In order to derive upper and lower bounds for $b_{1}(1)$, we can proceed in an analogous manner, only now we need upper and lower bounds on $\partial_{x} T_{P, l}$. Using (3.4), one may readily show that

$$
\partial_{x} T_{P, l}=\frac{T_{P, l}}{R^{l}(1)}\left[1+\sum_{n=1}^{\infty} R^{n l}(1) \partial_{x} T_{P, n i}\right]
$$

As shown in the Appendix, one has

$$
\partial_{x} T_{P, l}>\frac{T_{P, l}}{R^{\prime}(1)}
$$

and

$$
\partial_{x} T_{P, l}<\frac{T_{P, l}}{R^{l}(1)} \frac{1-R^{l}(1)}{1-R^{l}(1)-T_{P, l}}
$$

Using (3.25) in combination with (3.14), one obtains after some calculation

$$
\frac{1}{2} b_{1}^{2}(1)>e^{1-R_{-}(1)} \frac{1-R_{-}(1)+R_{-}^{2}(1)}{\left[1-R_{-}(1)\right]^{2}}=3.422654 \ldots
$$

giving $b_{1}(1)>2.616354 \ldots$ as lower bound and, somewhat more difficult,

$$
\begin{aligned}
\frac{1}{2} b_{1}^{2}(1) & <\frac{1}{R_{-}(1)}\left[1+\sum_{n=2}^{\infty} \frac{e^{-n}\left[1-R_{-}(1)\right]}{1-R_{+}^{n}(1)-e^{-n}}\right] \\
& <\frac{1}{R_{-}(1)}\left[1+\frac{e}{(e-1)\left\{e^{2}\left[1-R_{+}^{2}(1)\right]-1\right\}}\right]
\end{aligned}
$$

Evaluating the summation in $(3.28)$ results in $b_{1}(1)<2.719805 \ldots$, whereas (3.29) gives $b_{1}(1)<2.89074 \ldots$. The relative deviation between the upper and lower bounds is about 0.04 , so it is quite accurate [the exact value found by Otter is $b_{1}(1)=2.681127 \ldots$, which differs from the mean of (3.27), (3.28), yielding $b_{1}(1)=2.668079 \ldots$ by only about 0.005 as relative error].

With the specification of $R(1)$ and $b_{1}(1)$, the complete asymptotic behavior is known. We turn to the consequences for $T_{R}$ and $T$ next. Since 
$T_{R}=T_{P} / x$, we find, after expanding $1 / x$ to first order in the neighborhood of $x=R(y)$,

$$
T_{R}(x, y)=\frac{1}{R(y)}-\frac{b_{1}(y)}{R(y)} \Delta^{1 / 2}+\left(\frac{b_{2}(y)}{R(y)}+\frac{1}{R^{2}(y)}\right) \Delta+\cdots
$$

where we put $A=R(y)-x$. Inserting this into (3.8), one finds after an elementary calculation

$$
T(x, y)=C_{0}(y)+C_{2}(y) \Delta+C_{3}(y) \Delta^{3 / 2}+\cdots
$$

where

$$
C_{3}(y)=\frac{b_{1}(y) b_{2}(y)}{R(y)}=\frac{1}{3} \frac{b_{1}^{3}(y)}{R(y)}
$$

The main difference between the asymptotic behavior of $T$ in comparison with that of $T_{P}$ and $T_{R}$ is that the generating function for unrooted trees $T$ does not have the square root singular term; the derivative with respect to $x$ at $x=R(y)$ is finite. This difference implies that

$$
\begin{aligned}
T_{k, k+1} y^{k+1} & \approx \frac{3 C_{3}(y)}{4 \sqrt{\pi}} R(y)^{-k+3 / 2} k^{-5 / 2} \\
& =\frac{b_{1}^{3}(1) R^{3 / 2}(1)}{4 \sqrt{\pi}} R(1)^{-(k+1)} k^{-5 / 2} y^{k+1}
\end{aligned}
$$

so $T_{k, k+1} \sim R(1)^{-(k+1)} k^{-5 / 2}$, showing that the number of unrooted trees is much smaller than the number of rooted trees. In fact, $T_{k, k+1} \sim T_{k, k+1}^{R} / k$, which, roughly speaking, expresses the fact that almost all large rooted trees have no symmetries and so every vertex yields a different rooted tree.

In the next section we use the asymptotic results obtained above to analyze the polymer density dependence of the size distribution and the average number of polymers per complex. Also, it will be shown that the system undergoes a transition to a gel phase and the gelation boundary will be studied in some detail.

\section{STATISTICAL PROPERTIES OF THE COMPLEXES IN THE TREE APPROXIMATION}

In this section we first consider the asymptotic behavior of the size distribution and show that at sufficiently high polymer concentration the sytem undergoes a transition to a gel phase. Then we study the average number of block copolymers per complex. 
The equilibrium size distribution in the tree approximation is given by (2.13) and can be written using (3.33) as

$$
\gamma_{k}^{*}=v_{k}\left(h \alpha^{2} e^{-\lambda}\right)^{k} T_{k, k+1} \xi^{k+1} \approx \frac{\xi \chi}{R(1)} v_{k} k^{-5 / 2} z^{k}
$$

where

$$
z=\frac{h g_{1} \alpha e^{-\lambda}}{R(1)} ; \quad \chi=\frac{b_{1}^{3}(1) R^{3 / 2}(1)}{4 \sqrt{\pi}}
$$

The combinatorial factor $v_{k}$ was defined as $V / V_{k}$, in which $V_{k}$ is the average volume of a complex containing $k$ block copolymers. The size of a complex depends on the number of polymers in the complex, the size of a single polymer, and the average organization within the complex. We hypothesize that this volume $V_{k}$ obeys a scaling relation, and we put

$$
V_{k}=V_{\jmath} k^{\beta}
$$

In principle, the average volume of a complex with $k$ polymers also depends on the average number of domains. However, since the B parts are considered long and flexible, this volume will predominantly depend on $k$. Geometrically such a complex will resemble a sphere whose size is roughly independent of the number of domains. The value of $\beta$ is not known and we will treat it as a parameter in our model. A value of $\beta>0$ implies a decreasing translational combinatorial factor $v_{k}$. The volume $V_{0}$ is related to the volume of a single B part of a block copolymer in a good solvent. Since in a good solvent the polymers sense excluded volume effects, we have

$$
V_{0}=\frac{4}{3} \pi a^{3} M^{3 \mu}
$$

where $\mu \approx 3 / 5$ is Flory's constant, $a$ is the correlation length of the B parts, and $M$ is the number of segments of length $a$ constituting the B parts.

Hence, we have $v_{k}=v_{0} k^{-\beta}, v_{0} \equiv V / V_{0}$, and thus

$$
\gamma_{k}^{*}=\frac{\xi \chi \nu_{0}}{R(1)} k^{-(\beta+5 / 2)} z^{k}
$$

Since the asymptotic expression for $T_{k, k+1}$ gives a good approximation for the actual number of unrooted trees with $k$ edges also if $k$ is small, we will use it for all $k$. The distribution shows that larger complexes are always less likely; crucial is the long tail in the distribution if $z$ is close to 1 . We return to this momentarily. 
The value of $z$ is not free to choose, but follows from inserting $\gamma_{k}^{*}$ into the constraint (2.1), which results after some rewriting in

$$
\sum_{k=1}^{\infty} k^{-(\beta+3 / 2)} z^{k}=\frac{N R(1)}{\xi \chi v_{0}}=\frac{R(1)}{\chi} \frac{V_{0} \rho}{\xi} \equiv \rho^{*}
$$

where $\rho=N / V$ is the polymer density. The parameter $\xi$ is a measure for the likelihood that the A parts cluster. If $\xi$ decreases, this likelihood increases, since the clustering energy gain $U_{1}$ increases and/or the temperature decreases. However, as $\xi$ decreases, the right-hand side in (4.6) increases and consequently $z$ increases, resulting in more, larger complexes, in view of (4.5). It is not possible to express the left-hand side of (4.6) in closed form; hence, we can obtain $z$ only by numerical means as a function of $\rho^{*}$. If $\rho^{*} \ll 1$, one finds from (4.6) that

$$
z=\rho^{*}-2^{-(\beta+3 / 2)}\left(\rho^{*}\right)^{2}+\cdots
$$

A central property of the series in (4.6) is that it has a convergence radius equal to 1 and at $z=1$ the series is bounded. On the other hand, the right-hand side in (4.6) is experimentally controllable and can be made arbitrarily high, for instance, by adding more and more polymers and/or by decreasing the temperature, which causes $\xi$ to decrease. Thus, (4.6) has a solution $z<1$ if

$$
\rho^{*}<\rho_{c}^{*}(\beta) \equiv \sum_{k=1}^{\infty} k^{-(\beta+3 / 2)}
$$

If $\rho^{*}$ is larger than $\rho_{c}^{*}(\beta)$, then (4.6) does not have a solution and the size distribution $\Gamma^{*}$ is not defined. Hence, there is no solution in which all polymers can be grouped in complexes. A macroscopic gel is formed. Using (4.8), one readily shows that $\rho_{c}^{*}$ is a decreasing function of $\beta$ bounded by $\rho_{c}^{*}(0)=2.6 \ldots$ and 1 (the limiting value as $\beta \rightarrow \infty$ ). In Fig. 5 we plot the critical volume fraction $\left(V_{0} \rho\right)_{c}$ as a function of $\beta$ and $\xi$. Notice that if $\xi$ decreases, i.e., the likelihood of domains to be formed increases, gelation occurs at lower volume fractions. If $\xi$ is smaller than 0.25 approximately, a gel region exists for all $\beta$ values. Finally, the critical volume fraction is typically $O\left(10^{-2}\right)$ in experimental situations, hence $\xi=O\left(10^{-3}\right)$, as may be inferred from Fig. 5.

As an application, we consider $\langle k\rangle$, that is, the average number of block copolymers per complex. This is defined as

$$
\langle k\rangle=\frac{\sum_{k=1}^{\infty} k \gamma_{k}^{*}}{\sum_{k=1}^{\infty} \gamma_{k}^{*}}=\frac{\sum_{k=1}^{\infty} k^{-(\beta+3 / 2)} z^{k}}{\sum_{k=1}^{\infty} k^{-(\beta+5 / 2)} z^{k}}
$$




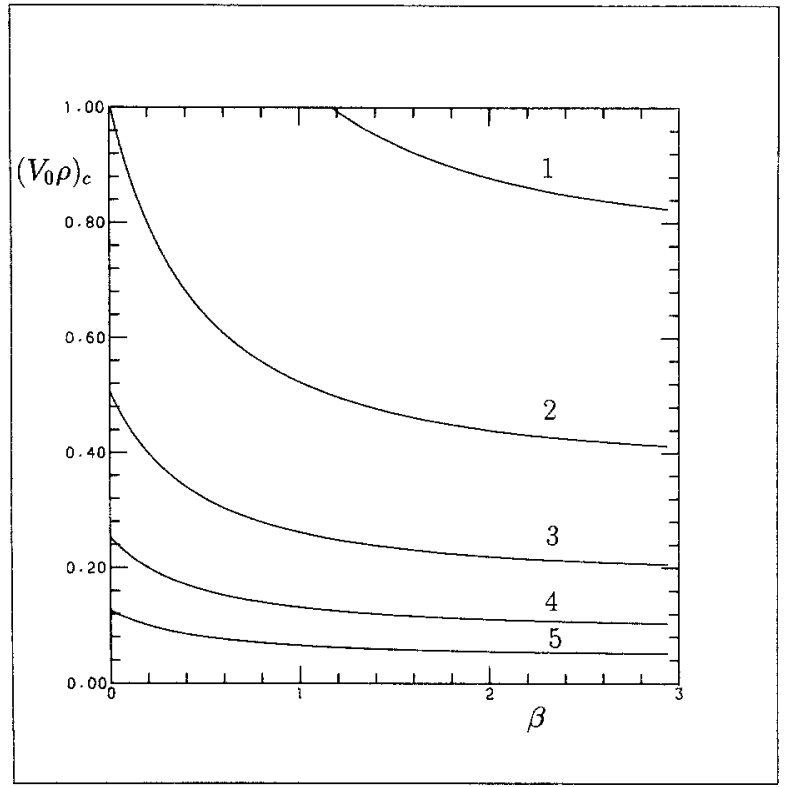

Fig. 5. The gelation boundary $\left(V_{0} \rho\right)_{c}$ as a function of $\beta$ at various $\xi$ values. The gel region is on the upper right-hand side of the curves. The value of $\xi$ used is given by $2^{-J}$; we indicate $j$ near the corresponding curve.

If $\rho^{*} \ll 1$, i.e., $z$ given by $(4.7)$ we find

$$
\langle k\rangle=1+2^{-(\beta+5 / 2)} \rho^{*}+2^{-2(\beta+5 / 2)}\left(\rho^{*}\right)^{2}+\cdots
$$

Hence, as $\beta$ increases, the average number of polymers in a complex decreases. We plot $\langle k\rangle$ as a function of $\rho^{*}$ in Fig. 6 for a few choices of $\beta$. Notice that the maximum of $\langle k\rangle$ decreases as $\beta$ increases and it tends to 1 if $\beta \rightarrow \infty$.

In the next section we extend the above treatment and include simple cycles into the structures.

\section{COUNTING LINE-DRESSED TREES}

In this section we extend the treatment given in Sections 3 and 4 to the case in which the complexes can be mapped to line-dressed trees. Line-dressed trees have a treelike topology; however, every edge in a line-dressed tree corresponds to an arbitrary number of block copolymers. Hence, simple cycles are included in the model, i.e., extra edges appear connecting two neighboring vertices on the treelike backbone. We first 


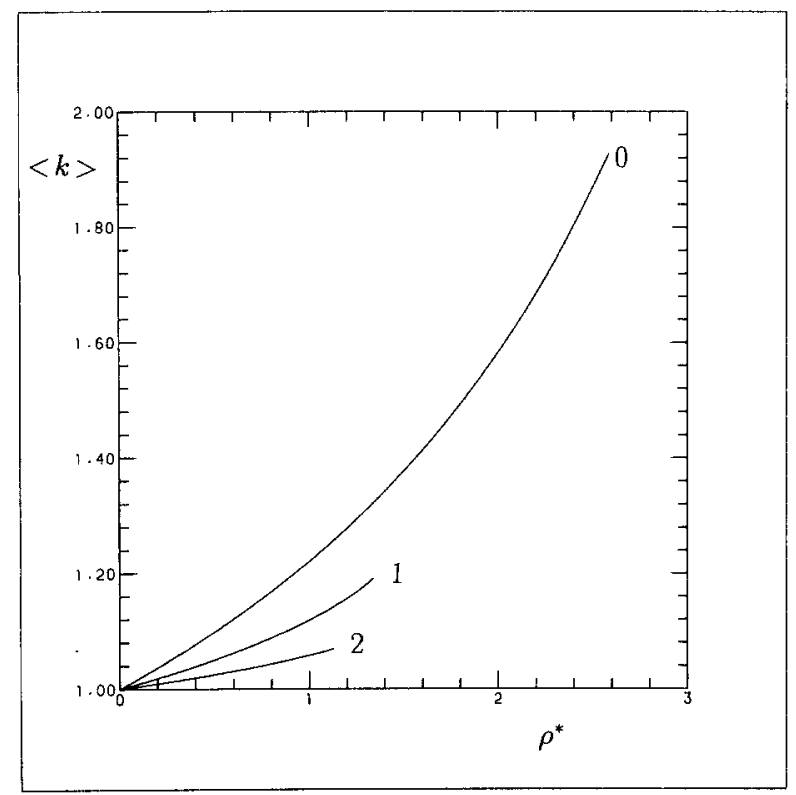

Fig. 6. The average number of polymers in a complex $\langle k\rangle$ as a function of $\rho^{*}$. The index near the curves indicates the corresponding $\beta$ value used.

derive functional equations for the generating functions for line-dressed trees. Then we study the singular behavior of these functions and finally we consider the consequences for the size distribution and the average number of polymers in a complex.

In Fig. 7 we have drawn the diagram for a dressed line, which is represented by a wiggly line. Let $f(x)$ denote the generating function for a dressed line; then we obtain from Fig. 7

$$
f(x)=x+x^{2}+x^{3}+\cdots=x /(1-x)
$$

Let $t_{R}(x, y)$ and $t_{P}(x, y)$ denote the generating functions for rooted and planted line-dressed trees, respectively. The diagram for rooted line-dressed trees is the same as in Fig. 2, provided one replaces all straight edges by wiggly lines. One thus obtains

$$
t_{R}(x, y)=y \exp \left(\sum_{l=1}^{\infty} \frac{t_{P}\left(x^{l}, y^{l}\right)}{l}\right)
$$

and

$$
t_{P}(x, y)=\frac{x}{1-x} t_{R}(x, y)=\frac{x y}{1-x} \exp \left(\sum_{l=1}^{\infty} \frac{t_{P}\left(x^{l}, y^{l}\right)}{l}\right)
$$




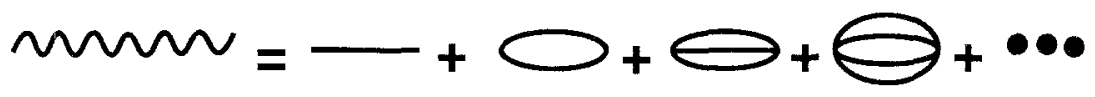

Fig. 7. Diagram for a dressed edge (wiggly line). Each subsequent diagram on the right-hand side consists of the previous one with one line added.

Following a similar argument as in Section 3, we may derive for the generating function for unrooted line-dressed trees $t(x, y)$

$$
t(x, y)=t_{R}(x, y)-\frac{1}{2} \frac{x}{1-x}\left[t_{R}^{2}(x, y)-t_{R}\left(x^{2}, y^{2}\right)\right]
$$

Using the manipulation program REDUCE, we find for the first few terms of $t_{R}$ and $t$

and

$$
\begin{aligned}
t_{R}(x, y)= & y+\sum_{k=1}^{\infty} \sum_{m=2}^{k+1} t_{k, m}^{R} x^{k} y^{m} \\
= & y+x y^{2}+x^{2}\left(y^{2}+2 y^{3}\right) \\
& +x^{3}\left(y^{2}+3 y^{3}+4 y^{4}\right)+x^{4}\left(y^{2}+5 y^{3}+9 y^{4}+9 y^{5}\right) \\
& +x^{5}\left(y^{2}+6 y^{3}+18 y^{4}+26 y^{5}+20 y^{6}\right)+\cdots
\end{aligned}
$$

$$
\begin{aligned}
t(x, y)= & y+\sum_{k=1}^{\infty} \sum_{m=2}^{k+1} t_{k, m} x^{k} y^{m} \\
= & y+x y^{2}+x^{2}\left(y^{2}+y^{3}\right) \\
& +x^{3}\left(y^{2}+y^{3}+2 y^{4}\right)+x^{4}\left(y^{2}+2 y^{3}+3 y^{4}+3 y^{5}\right) \\
& +x^{5}\left(y^{2}+2 y^{3}+6 y^{4}+6 y^{5}+6 y^{6}\right)+\cdots
\end{aligned}
$$

In order to get an impression of the number of structures with $k$ block copolymers, we also have

$$
t_{R}(x, 1)=1+x+3 x^{2}+8 x^{3}+24 x^{4}+71 x^{5}+224 x^{6}+710 x^{7}+2318 x^{8}+\cdots
$$

and

$$
t(x, 1)=1+x+2 x^{2}+4 x^{3}+9 x^{4}+21 x^{5}+55 x^{6}+146 x^{7}+415 x^{8}+\cdots
$$


We have drawn all rooted and unrooted line-dressed trees with up to three edges in Fig. 8. It is left to the reader to verify (5.5), (5.6) using this figure.

We now turn to the singular behavior and consider $t_{P}(x, y)$ first. Consequences for $t_{R}(x, y)$ and $t(x, y)$ will be determined afterward. Let $r(y)$ denote the convergence radius of $t_{P}(x, y)$. Clearly, $t_{P}(x, y)$ is an analytic function of $x$ if $|x|<r(y)$. Consider

$$
\mathscr{G}(\zeta, x, y)=\frac{x y}{1-x} \exp \left(\zeta+\sum_{l=2}^{\infty} \frac{t_{P}\left(x^{l}, y^{l}\right)}{l}\right)-\zeta
$$

Then $\mathscr{G}_{(}\left(t_{P}(x, y), x, y\right)=0$ and from the condition $\mathscr{G}_{\xi}\left(t_{P}(r(y), y), r(y), y\right)$ $=0$ describing the singular point, we find

$$
t_{P}(r(y), y)=1
$$

Since $\mathscr{G}_{\zeta \zeta}\left(t_{P}(r(y), y), r(y), y\right)=1$, we have the expansion

$$
t_{P}(x, y)=1-a_{1}(y) \Delta^{1 / 2}+a_{2}(y) \Delta+a_{3}(y) \Delta^{3 / 2}+\cdots
$$

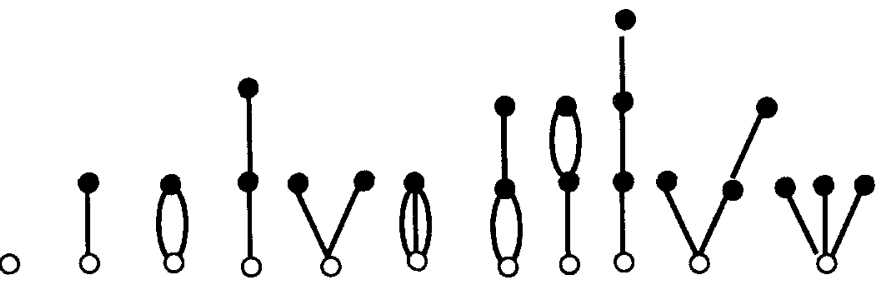

(a)
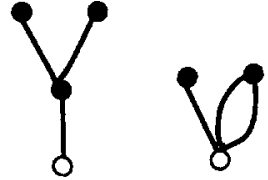

(b)

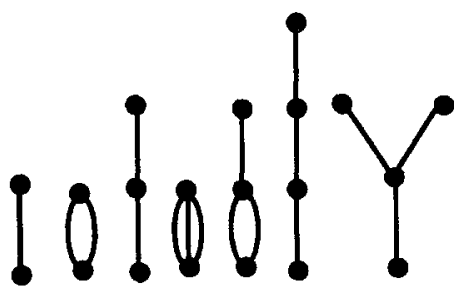

Fig. 8. All (a) rooted and (b) unrooted line-dressed trees with up to three edges. 
which is valid for $x$ smaller than but close to $r(y)$. After some calculation one may show that

$$
\frac{1}{2} a_{1}^{2}(y)=\frac{1}{r(y)}\left[\frac{1}{1-r(y)}+\sum_{l=2}^{\infty} r^{l}(y) \partial_{x} t_{P}\left(r^{l}(y), y^{\prime}\right)\right]
$$

and

$$
a_{2}(y)=\frac{1}{2} a_{1}^{2}(y)
$$

We derive precise upper and lower bounds on $r(y)$ and $a_{1}(y)$ in order to fully specify the asymptotic behavior of the number of planted line-dressed trees, given by

$$
\sum_{m=1}^{k} t_{k, m}^{P} y^{m} \approx \frac{a_{1}(y) r(y)^{1 / 2}}{2 \sqrt{\pi}} r^{-k}(y) k^{-3 / 2}
$$

Since $t_{P}(r(y), y)=1$, we find from (5.3)

$$
\frac{y r(y)}{1-r(y)}=e^{-1} \prod_{n=2}^{\infty} e^{-t_{P, n} / n}
$$

where we put $t_{P, n}=t_{P}\left(r^{n}(y), y^{n}\right)$. Solving $r(y)$ from this gives

$$
r(y)=[1+e y \mathscr{A}(y)]^{-1} ; \quad \mathscr{A}(y)=\prod_{n=2}^{\infty} e^{t_{p, n} / n}
$$

Using the upper bound $t_{P, n}<e^{-n}$ as shown in the Appendix, we obtain

$$
r(y)>r_{-}(y) \equiv\left[1+\frac{e y}{e-1} \exp \left(\frac{e-1}{e}\right)\right]^{-1}
$$

The lower bound $t_{P, n}>\left[y r_{-}(y)\right]^{n}$ results in

$$
r(y)<r_{+}(y) \equiv\left[1+y \frac{\exp \left[1-y r_{-}(y)\right]}{1-y r_{-}(y)}\right]^{-1}
$$

The maximal relative deviation between the upper and lower bounds is about 0.04 for $0 \leqslant y \leqslant 1$. In the following we will use the mean value of the upper and lower bounds as our estimate for $r(y)$. Notice that $r(y)$ is a decreasing function of $y$ and for small $y$ we have $r(y) \approx 1-e y$ to a good approximation [in fact, we used $\exp (-1 / e) \approx(e-1) / e]$. finds

Next, we consider upper and lower bounds for $a_{1}(y)$. Using (5.3), one

$$
\partial_{x} t_{P, l}=\frac{t_{P, l}}{r^{l}(y)}\left[\frac{1}{1-r^{l}(y)}+\sum_{n=2}^{\infty} r^{n l}(y) \partial_{x} t_{P, n l}\right]
$$


and since $\partial_{x} t_{P, n l}>0$, we have

$$
\partial_{x} t_{P, l}>\frac{t_{P, l}}{r^{l}(y)\left[1-r^{l}(y)\right]}
$$

which implies after some calculation

$$
\frac{1}{2} a_{1}^{2}(y)>\frac{1}{r_{+}(y)}\left[\frac{1}{1-r_{-}(y)}+\frac{\left[y r_{-}(y)\right]^{2}}{1-y r_{-}(y)}\right]
$$

Finally, since $\partial_{x} t_{P, l}>\partial_{x} t_{P, n l}, n=2,3, \ldots$, one obtains, using (5.19),

$$
\partial_{x} t_{P, l}<\frac{t_{P, l}}{r^{l}(y)} \frac{1}{1-r^{l}(y)-t_{P, l} r^{l}(y)}
$$

resulting in the estimate

$$
\frac{1}{2} a_{1}^{2}(y)<\frac{1}{r_{-}(y)}\left[\frac{1}{1-r_{+}(y)}+\sum_{n=2}^{\infty} \frac{e^{-n}}{1-r_{+}^{n}(y)\left(1+e^{-n}\right)}\right]
$$

This estimate is valid if $r_{+}^{2}(y)<1 /\left(1+e^{-2}\right)$ or $y>e^{-3} / 2$ approximately. The maximal deviation in $a_{1}(y)$ as given by $(5.21),(5.23)$ is very small if $y>e^{-3 / 2}$. Hence, we use $a_{1}(y)$ as given by (5.21) for all $y>0$, avoiding the complications with the upper bound (5.23). If $y \ll 1$, then $a_{1}(y) \approx(2 / e y)^{1 / 2}$. In comparison with the normal tree case, we notice that $r(y)$ is bounded from above, whereas $R(y) \sim y^{-1}$, and $a_{1}(y) \sim y^{-1 / 2}$, whereas $b_{1}(y) \sim y^{1 / 2}$.

We now determine the singular behavior of $t_{R}$ and $t$. If we expand $(1-x) / x$ to first order around $x=r(y)$, and using (5.2), we find

$$
\begin{aligned}
t_{R}(x, y)= & \frac{1-r(y)}{r(y)}-\frac{a_{1}(y)[1-r(y)]}{r(y)} \Delta^{1 / 2} \\
& +\left(\frac{a_{2}(y)[1-r(y)]}{r(y)}+\frac{1}{r^{2}(y)}\right) \Delta+\cdots
\end{aligned}
$$

Inserting this into (5.4) and ordering like powers of $\Delta$, we find for $t(x, y)$

$$
t(x, y)=c_{0}(y)+c_{2}(y) \Delta+c_{3}(y) \Delta^{3 / 2}+\cdots
$$

where

$$
c_{3}(y)=\frac{a_{1}(y) a_{2}(y)[1-r(y)]}{r(y)}=\frac{1}{3} \frac{a_{1}^{3}(y)[1-r(y)]}{r(y)}
$$


which implies

$$
\sum_{m=2}^{k+1} t_{k, m} y^{m} \approx \frac{a_{1}^{3}(y)[1-r(y)] r^{3 / 2}(y)}{4 \sqrt{\pi}} r(y)^{-(k+1)} k^{-5 / 2}
$$

So, just as in the case of normal trees, we obtain a $-5 / 2$ power law dependence.

Using this asymptotic behavior, we find for the size distribution

$$
\gamma_{k}^{*} \approx \frac{\chi(\xi) v_{0}}{r(\xi)} k^{-(\beta+5 / 2)} \tilde{z}^{k}
$$

where

$$
\tilde{z} \equiv \frac{h \alpha^{2} e^{\lambda}}{r(\xi)} ; \quad \chi(\xi) \equiv \frac{a_{1}^{3}(\xi)[1-r(\xi)] r(\xi)^{3 / 2}}{4 \sqrt{\pi}}
$$

Notice that $\chi(\xi) \approx(1 / \pi \xi)^{1 / 2}$ if $\xi \ll 1$. Hence, for small $\xi$ the number of complexes increases very rapidly if $\xi$ decreases. The singularity in $\chi(\xi)$ influences essentially the physical properties of the complexes. The variable $\tilde{z}$ follows from inserting (5.28) into (2.1) and yields the equation

$$
\sum_{k=1}^{\infty} k^{-(\beta+3 / 2)} \tilde{z}^{k}=\frac{N r(\xi)}{\chi(\xi) v_{0}}=\frac{r(\xi)}{\chi(\xi)} V_{0} \rho \equiv \tilde{\rho}
$$

This equation has the same qualitative aspects as we found in the previous section. In order to compare these two cases, we must relate $\rho^{*}$ and $\tilde{\rho}$. We have

$$
\tilde{\rho}=\frac{\chi \xi}{\chi(\xi)} \frac{r(\xi)}{R(1)} \rho^{*}
$$

An interesting consequence of the dressing of the lines is that no gel region exists. That is, for all $\xi$ and $\beta$ values the critical volume fraction is 1 . Hence, the topological structures accessible to the complexes have an essential influence on the physical properties of the system. Since this assumption usually is made ad hoc, we emphasize that due care should be taken as regards to this assumption in similar studies.

The expression for $\langle k\rangle$ is the same as in Eq. (4.11) with $\tilde{z}$ replacing $z$. In view of (5.31) the density dependence of $\langle k\rangle$ is, however, quite different. We plot $\langle k\rangle$ as a function of $\rho^{*}$ in Fig. 9. Compared to the normal tree case, we notice that $\langle k\rangle$ is smaller at the same $\rho^{*}$ and decreases as $\xi$ decreases. This is related to the extra structures accessible due to the dressing of the lines, resulting in more smaller complexes.

In the next section we determine the relative increase in viscosity and compare the results obtained for normal and line-dressed trees. 


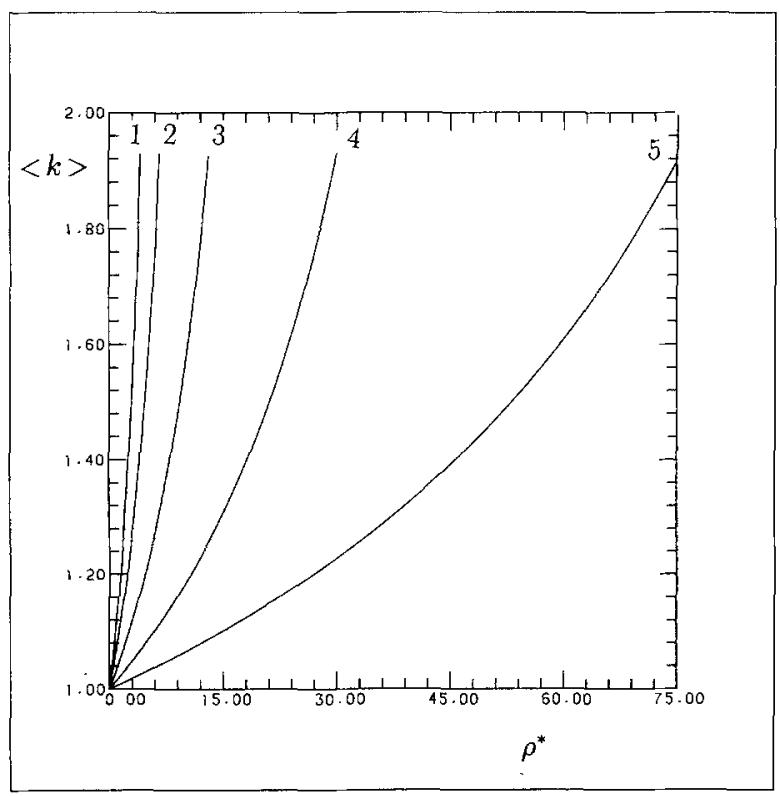

Fig. 9. The average number of block copolymers per complex as a function of $\rho^{*}$. We use $\beta=0$ and $\xi=2^{-j}$, with $j$ as index near the corresponding curve.

\section{CALCULATION OF THE RELATIVE CHANGE IN VISCOSITY}

First, we express the relative change in viscosity in terms of the size distribution of the complexes. Then we show the dependence of the viscosity on polymer density.

Let $\eta_{0}$ denote the viscosity of the solvent and $\eta$ the viscosity of the solution containing block copolymer complexes. As was shown by Felderhof, ${ }^{(8)}$ the relative change in viscosity for a dilute solution of porous spheres of radius $r$ is given by

$$
\frac{\eta-\eta_{0}}{\eta_{0}}=\frac{10}{3} \pi \rho_{p} r^{3} \frac{H\left(\sigma_{r}\right)}{1+10 H\left(\sigma_{r}\right) / \sigma_{r}^{2}}
$$

where $\rho_{p}$ is the sphere density and

$$
H(x)=1+\frac{3}{x^{2}}-\frac{3}{x} \operatorname{coth}(x)
$$

Furthermore, $\sigma_{r}=\kappa r$, where $\kappa^{2}=\phi \rho^{\prime} / \eta_{0}$, in which $\phi$ is the friction coefficient of the segments of the molecules and $\rho^{\prime}$ the segment density in 
the porous sphere. Hydrodynamic interactions between the spheres are disregarded in (6.1).

We now derive an equation expressing the relative change in viscosity for the system of block copolymer complexes. Since the B parts of the block copolymers are much larger than the A parts, any complex will geometrically resemble a "cloud" of B-type material predominantly. We disregard the different permeability of the domains as far as their contribution to the increase in viscosity is concerned ${ }^{(9)}$ and treat the complexes as uniform porous spheres. The system can be regarded as a mixture of porous spheres of various sizes, which implies

$$
\frac{\eta-\eta_{0}}{\eta_{0}}=\frac{10}{3} \pi \sum_{k=1}^{\infty} \rho_{p, k} r_{k}^{3} \frac{H\left(\sigma_{k}\right)}{1+10 H\left(\sigma_{k}\right) / \sigma_{k}^{2}}
$$

where $\rho_{p, k}$ is the density of complexes containing $k$ polymers, i.e.,

$$
\rho_{p, k}=\gamma_{k}^{*} / V
$$

Also, $r_{k}$ is a measure for the size of the complexes containing $k$ polymers: one has the relation $V_{k}=4 \pi r_{k}^{3} / 3$. In view of (4.3), (4.4), one thus obtains

$$
r_{k}^{3}=\frac{3}{4 \pi} V_{0} k^{\beta}=a^{3} M^{3 \mu} k^{\beta}
$$

We finally specify $\sigma_{k}$. The segment density inside a complex with $k$ polymers is given by

$$
\rho_{k}^{\prime}=\frac{M k}{V_{k}}=\frac{M}{V_{0}} k^{1-\beta}
$$

Hence, we find after some rewriting

$$
\sigma_{k}=\left(\frac{3 \phi M^{1-\mu}}{4 \pi a \eta_{0}}\right)^{1 / 2} k^{(1-\beta / 3) / 2} \equiv \mathscr{B} k^{(1-\beta / 3) / 2}
$$

We notice that, since $\beta<3, \sigma_{k} \rightarrow \infty$ as $k \rightarrow \infty$, i.e., larger complexes are less permeable. A remark is in order as regards (6.3). Following Felderhof, ${ }^{(8)}$ we disregard hydrodynamic interactions between the complexes. Hence, all spheres can be regarded as independent. This implies that (6.3) is only valid in the dilute regime, strictly speaking. We will, however, use it invariably for all densities up to the gelation transition in order to get an order-of-magnitude estimate for the change in viscosity due to an increase in polymer density and/or temperature. We now consider the two cases treated. 
For normal trees we derived $\gamma_{k}^{*}$ as given in Eq. (4.1) and after some rewriting we find

$$
\frac{\eta-\eta_{0}}{\eta_{0}}=\frac{5}{2} \frac{\xi \chi}{R(1)} \sum_{k=1}^{\infty} z^{k} k^{-5 / 2} \frac{H\left(\sigma_{k}\right)}{1+10 H\left(\sigma_{k}\right) / \sigma_{k}^{2}}
$$

where use was made of (4.3) and the definition of $v_{k}$. For line-dressed trees we obtain an expression similar to $(6.8)$ with $\chi(\xi) / r(\xi)$ replacing $\xi \chi / R(1)$. We will study the impermeable sphere limit $(\mathscr{B} \gg 1)$ and the free draining limit $(\mathscr{B} \ll 1)$ first and then present numerical examples for the increase in viscosity. If $\mathscr{B} \gg 1$, then obviously $\sigma_{k} \gg 1$ and hence $H\left(\sigma_{k}\right) \approx 1 ; k=1,2, \ldots$. In the dilute regime, i.e., $\rho^{*} \ll 1$, the series in (6.8) is well approximated by the first two terms, so for normal trees one obtains

$$
\frac{\eta-\eta_{0}}{\eta_{0}} \approx \frac{5}{2} \frac{\xi \chi}{R(1)}\left(z+2^{-5 / 2} z^{2}\right)
$$

Combination with (4.7) yields, up to second order in $\rho^{*}$,

$$
\begin{aligned}
\frac{\eta-\eta_{0}}{\eta_{0}} & =\frac{5}{2} \frac{\xi \chi}{R(1)}\left[\rho^{*}+2^{-5 / 2}\left(\rho^{*}\right)^{2}\left(1-2^{-(\beta-1)}\right)\right] \\
& =\frac{5}{2} V_{0} \rho+\frac{5}{2} 2^{-5 / 2}\left[1-2^{-(\beta-1)}\right] \frac{R(1)}{\xi \chi}\left(V_{0} \rho\right)^{2}+\cdots ; \quad \mathscr{B} \gg 1
\end{aligned}
$$

The intrinsic viscosity $[\eta]$ is defined through

$$
\eta=\eta_{0}(1+[\eta] \rho+\cdots)
$$

Hence, the intrinsic viscosity is $5 V_{0} / 2$, a result already obtained by Einstein. Notice that the second-order coefficient in the hard-sphere limit is either positive or negative depending on $\beta$. If $\beta>1$, then it is positive and negative elsewhere. The corresponding expression for line-dressed trees is the same as given by $(6.10)$ upon replacing $R(1) / \xi \chi$ by $r(\xi) / \chi(\xi)$. In the free draining limit $\mathscr{B} \ll 1$ and hence $H\left(\sigma_{k}\right) \approx \sigma_{k}^{2} / 10$ for those terms in the series (6.8) that contribute most to the series. After some calculation one obtains in the dilute regime

$$
\frac{\eta-\eta_{0}}{\eta_{0}}=\frac{\mathscr{B}^{2}}{8} V_{0} \rho+\frac{\mathscr{B}^{2}}{8} \frac{R(1)}{\zeta \chi} 2^{-(\beta+3 / 2)}\left(2^{2 \beta / 3}-1\right)\left(V_{0} \rho\right)^{2}+\cdots ; \quad \mathscr{B} \ll 1
$$

for normal trees. The corresponding expression for line-dressed trees follows upon the same replacements as mentioned above. Notice that the 
second-order coefficient is always positive in this regime. It must be stressed that the second-order coefficient cannot be identified with Huggins' coefficient; it merely expresses the influence of variations in the complex distribution on the viscosity, disregarding hydrodynamic interactions.

We next present the results obtained from a numerical determination of $z(\tilde{z})$ from (4.6) [(5.30)] as a function of $\rho^{*}(\tilde{\rho})$. In Fig. 10 we present the results for normal trees. These results are qualitatively in agreement with (6.10), i.e., all results resemble the hard-sphere case. If $\beta<1$, the increase in viscosity decreases towards gelation, and if $\beta>1$, this behavior is reversed. Also, the increase is more pronounced if $\mathscr{B}$ is larger, consistent with $(6.8)$ and the behavior of $H\left(\sigma_{k}\right)$.

Finally, in Fig. 11 we show some typical results for line-dressed trees. We use $\beta=0$ and concentrate on variations in $\xi$. Notice that if $\xi$ decreases, the viscosity increases, just as one may expect. Again, this increase is more pronounced if $\mathscr{B}$ is larger. Furthermore, if $\xi$ is chosen small enough, the increase in viscosity can be made arbitrarily high. If one were to consider partial line-dresed trees, i.e., trees in which every edge corresponds to up to a maximal number of block copolymers, then one could choose variables

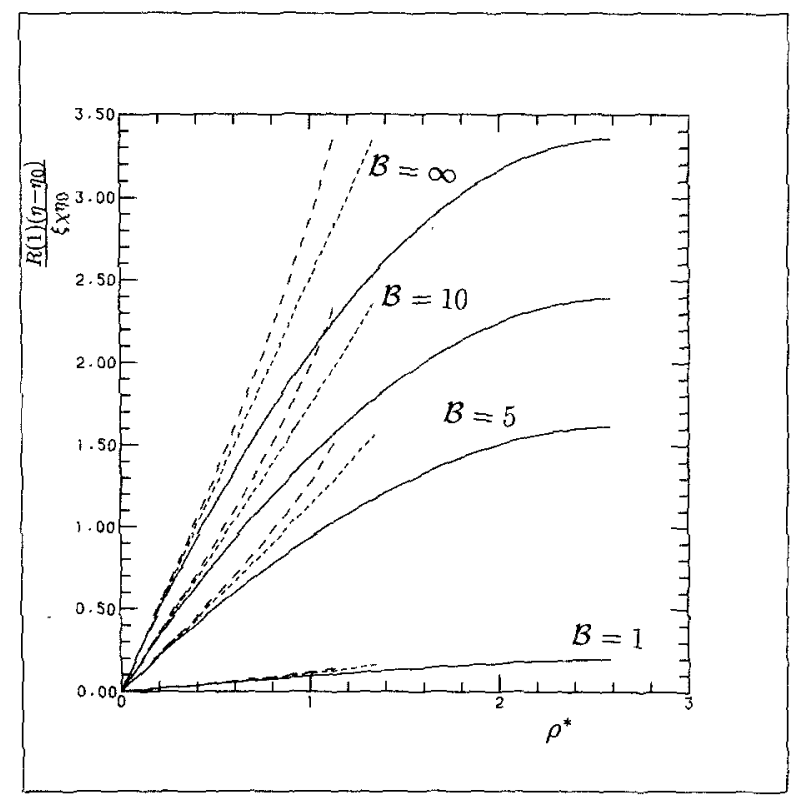

Fig. 10. The increase in viscosity as a function of density for normal trees. We plot $R(1)\left(\eta-\eta_{0}\right) / \zeta \chi \eta_{0}$ versus $\rho^{*}$. The solid curves correspond to $\beta \approx 0$, the short-dashed curves to $\beta=1$, and the long-dashed curves to $\beta=2$. The values for $\mathscr{B}$ used are indicated near the corresponding set of curves. 


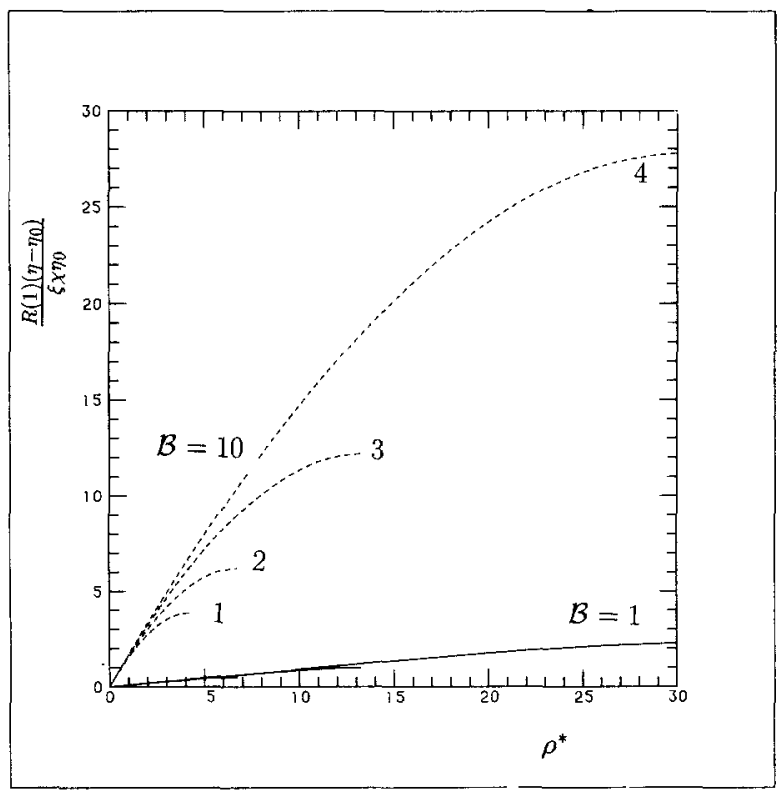

Fig. 11. Plot of $R(1)\left(\eta-\eta_{0}\right) / \xi \chi \eta_{0}$ versus $\rho^{*}$ for line-dressed trees. We use $\beta=0$ and indicate the values of $\mathscr{B}$ and $\xi=2^{-j}$ used near the corresponding curves.

such that (a) gelation occurs and (b) the increase in viscosity can be made quite large, such as is observed in experimental situations. Presently, detailed experimental studies are performed relating to the temperature and density dependence of both intrinsic viscosity and the gelation transition of a specific "model system." (10)

\section{APPENDIX}

In this appendix we derive upper and lower bounds for the generating function for planted trees in the points $(x, y)=\left(R^{l}(y), y^{l}\right)$ for $l=2,3, \ldots$, both for the normal tree case and for line-dressed trees.

We consider first normal trees. Since

$$
T_{P}(x, y)=x y \exp \left[\sum_{l=1}^{\infty} \frac{T_{P}\left(x^{l}, y^{l}\right)}{l}\right]
$$

we have, using the notation $T_{P, l}=T_{P}\left(R^{I}(1), 1\right)$,

$$
T_{P, l}=R^{l}(1) e^{T_{P, l}} \prod_{n=2}^{\infty} e^{T_{P, n l / n}}
$$


so, solving $R(1)$ from this, we have

$$
R^{l}(1)=T_{P, l} e^{-T_{P, l}} \prod_{n=2}^{\infty} e^{T_{P, n} / n}
$$

Also, in view of (3.19) we have

$$
R^{\prime}(1)=e^{-l} \prod_{n=2}^{\infty} e^{-l T_{P, n} / n}
$$

so, combining Eqs. (A.3) and (A.4), we get

$$
T_{P, l} e^{-T_{P, l}}=e^{-l} \prod_{n=2}^{\infty} e^{-\left(l T_{P, n}-T_{P, n l}\right) / n}
$$

which can be rewritten as

$$
T_{P, l} e^{T_{P, l}}=e^{-l} e^{-T_{P, l}} \prod_{n=2}^{\infty} e^{-\left(I T_{P, n}-T_{P, n l}\right) / n}
$$

where the prime on the product in (A.6) indicates that $n \neq l^{j}, j=1,2, \ldots$. One may easily verify that $T_{P, n}-T_{P, n l}>0$, and so

$$
T_{P, l}<e^{-l}
$$

which proves (3.20). A lower bound on $T_{P, l}$ can be derived by using (A.6), which gives

$$
T_{P, l}>e^{-l} \prod_{n=2}^{\infty} e^{-l T_{P, n} / n}
$$

where use was made of $T_{P, n l}>0$. However, (A.8) implies

$$
T_{P, l}>e^{-l} \prod_{n=2}^{\infty} e^{-l T_{P_{1}, n}}=R^{l}(1)>R_{-}^{l}
$$

proving (3.22). Next we consider upper and lower bounds on $\partial_{x} T_{p_{1}, l}$. We have, using (3.24), and the fact that $\partial_{x} T_{P, l}>0$,

$$
\partial_{x} T_{P, l}>\frac{T_{P, l}}{R^{I}(1)}
$$

proving (3.25). Also, it can be easily verified that $\partial_{x} T_{P, l} \geqslant \partial_{x} T_{P, n l}$, $n=1,2, \ldots$, so

$$
\partial_{x} T_{P, l}<\frac{T_{P, l}}{R^{l}(1)}\left[1+\partial_{x} T_{P, l} \frac{R^{l}(1)}{1-R^{l}(1)}\right]
$$

Solving $\partial_{x} T_{P, l}$ from this results in (3.26). 
We next turn to line-dressed trees. The derivation is quite analogous to the one just given, and we only give a short sketch of it. The generating function for planted line-dressed trees is given by

$$
t_{P}(x, y)=\frac{x y}{1-x} \exp \left(\sum_{l=1}^{\infty} \frac{t_{P}\left(x^{l}, y^{l}\right)}{l}\right)
$$

so, using the notation $t_{P, l}=t_{P}\left(r^{l}(y), y^{l}\right)$, we have

$$
t_{P, l}=\frac{r^{l}(y) y^{l}}{1-r^{l}(y)} e^{t_{P, l}} \prod_{n=2}^{\infty} e^{t_{P, n} / n}
$$

We can solve $r^{l}(y)$ from this and equate it to the form given in (5.16). After some rewriting, one finds that

$$
t_{P, l} e^{-t_{P, l}}<e^{-l} e^{-t_{P, l}} \prod_{n=2}^{\infty} e^{-\left(l_{P, n}-t_{P, n i}\right) / n}
$$

resulting in

$$
t_{P, l}<e^{-l}
$$

The lower bound can be derived straightforwardly, since

$$
t_{P, l}>t_{P, l} e^{-t_{P, l}}>\frac{y^{l} r^{l}(y)}{1-r^{l}(y)}>[y r-(y)]^{l}
$$

Finally, the upper and lower bounds on $\partial_{x} t_{P, I}$ can be derived following exactly the same steps as in the normal tree case. We will not repeat this here.

\section{REFERENCES}

1. R. van Damme and B. J. Geurts, J. Stat. Phys. $57: 1099$ (1989).

2. P. Harary and F. Palmer, Graphical Enumeration (Academic Press, 1973).

3. B. J. Geurts and R. van Damme, 5th IFF-ILL Workshop, Julich, to appear (1988).

4. J. Batoulis and K. Kremer, Preprint, Universität Mainz (1988).

5. F. W. Wiegel and A. S. Perelson, J. Stat. Phys. 29:813 (1982).

6. A. C. Hearn, REDUCE User's Manual (Rand, 1983).

7. R. Otter, Ann. Math. 49:583 (1948).

8. B. U. Felderhof, Physica 80A:174 (1975).

9. F. W. Wiegel, Fluid Flow Through Porous Macromolecular Systems (Springer-Verlag, 1980).

10. K. Visscher and P. F. Mijnlieff, In preparation (1989). 